\title{
COMPARAÇÃO ENTRE RESSECÇÃO COM ANASTOMOSE PRIMÁRIA E RESSECÇÃO EM ESTÁGIOS NOS TUMORES OBSTRUTIVOS DO CÓLON ESQUERDO
}

\author{
José Eduardo de AGUILAR-NASCIMENTO, Cervantes CAPOROSSI e \\ Mariana NASCIMENTO
}

\begin{abstract}
RESUMO - Racional - A melhor opção no tratamento cirúrgico do câncer obstrutivo do cólon esquerdo ainda é controversa. Objetivo - Relatar a experiência de três hospitais de Cuiabá, MT, no tratamento da obstrução neoplásica do cólon esquerdo, enfocando os resultados imediatos com a ressecção e anastomose primária e com a ressecção em estágios. Casuística e Métodos - Estudaram-se retrospectivamente 23 pacientes (idade mediana $=52$ [39-84] anos) com adenocarcinomas ressecáveis obstrutivos do cólon esquerdo, sendo $10(43,5 \%)$ do gênero masculino e 13 (56,5\%) do feminino. Em 14 (60,9\%), o tratamento foi realizado em um tempo, com ressecção e anastomose primária, e em $9(39,1 \%)$ realizou-se ressecção em estágios. Nos 14 pacientes submetidos a ressecção e anastomose primária, realizou-se ressecção segmentar com preparo intra-operatório do cólon $(\mathrm{n}=10)$ ou colectomia subtotal $(n=4)$. Nos doentes submetidos a ressecção em estágios, quatro casos foram submetidos a operação de Hartmann (ressecção em dois tempos) e cinco casos a colostomia em alça no ângulo hepático (ressecção em três tempos). Resultados - Dois pacientes $(8,7 \%)$ que pertenciam ao grupo da ressecção em estágios, faleceram no pós-operatório. Quatro $(44,4 \%)$ dos pacientes com ressecção em estágios não completaram o tratamento com o fechamento da colostomia. A incidência de complicações foi de $28,6 \%$ nos casos submetidos a ressecção e anastomose primária (4/14) e de $66,7 \%$ nos operados com a ressecção em estágios (6/9). Ocorreram três casos de fístula, sendo um caso $(7,1 \%)$ com a ressecção e a anastomose primária e dois $(22,2 \%)$ com a ressecção em estágios. Conclusãa - O conjunto dos resultados sugere que o tratamento em um tempo na obstrução do cólon esquerdo é seguro e pode ser indicado na maioria dos casos.
\end{abstract}

DESCRITORES - Obstrução intestinal. Neoplasias colorretais. Colectomia. Colostomia.

\section{INTRODUÇÃO}

Embora haja consenso de que o melhor tratamento na obstrução do cólon direito seja a ressecção da lesão seguida de anastomose primária, ainda existe controvérsia sobre a melhor forma de conduzir uma oclusão do lado esquerdo ${ }^{(7,14,18)}$. Anastomoses realizadas na vigência de obstrução distal têm sido associadas a maior risco de deiscência devido ao edema, distensão e dificuldade no manuseio cirúrgico do intestino grosso repleto de gases e fezes ${ }^{(1,11)}$. Por esta razão, alguns autores têm preferido o tratamento em estágios que envolvem, inicialmente, a realização de estoma, com ou sem ressecção da lesão obstrutiva, e a seguir, dependendo do que foi realizado, mais uma ou duas operações para a resolução completa do problema $^{(7,16,18)}$.

Esse tratamento em estágios, entretanto, não é isento de críticas. O número de atos operatórios é maior, parece não haver menor mortalidade, os custos são maiores, alguns pacientes não conseguem ser novamente operados para a ressecção do tumor ou para o fechamento do estoma e o tempo de inatividade é maior. Além disso, a condição de vida para aqueles casos paliativos é, certamente, $\operatorname{pior}^{(7,8,10,12,14)}$.

Trabalho realizado no Departamento de Cirurgia da Faculdade de Ciências Médicas da Universidade Federal de Mato Grosso. 
Por outro lado, há na literatura relatos em número cada vez maior, mostrando que a ressecção da lesão neoplásica associada à anastomose primária é possível, eficaz e com aceitáveis taxas de morbimortalidade. As principais opções para este tipo de operação em um só tempo são: a ressecção segmentar do cólon esquerdo associada ao preparo intraoperatório do cólon (PIOC) ${ }^{(2,5,6,8,9,13,14,19,22)}$ e a colectomia subtotal ou total seguida de ileossigmóide ou ileorretotostomia ${ }^{(4,10,12,14,23)}$. Entretanto, há carência de trabalhos controlados sobre esse tópico na literatura.

O objetivo deste trabalho é relatar a experiência do nosso grupo no tratamento da obstrução do cólon esquerdo devido ao câncer colorretal, enfocando os resultados imediatos com um só tempo pela ressecção e anastomose primária (RAP) e com a ressecção em estágios (RE).

\section{CASUÍSTICA E MÉTODO}

Foram revistos, retrospectivamente, os prontuários de 41 pacientes com obstrução neoplásica do cólon, atendidos e operados no período de janeiro de 1988 a dezembro de 1999 em um dos três hospitais onde os autores operam: Hospital Universitário Júlio Muller, Santa Casa de Cuiabá e Hospital Santa Rosa, Cuiabá, MT. Foram excluídos do estudo 18 (43,9\%) pacientes pelos seguintes motivos: a) obstrução localizada no cólon direito, definido como a porção do intestino grosso situada entre o ceco e até o ângulo esplênico $(\mathrm{n}=11)$, e b) neoplasia avançada sem possibilidade de ressecção no inventário da primeira operação $(n=7)$. Foram estudados, portanto, $23(56,1 \%)$ casos onde a obstrução era ressecável e localizava-se à esquerda. Obstrução colônica foi definida pela presença de distensão colônica na radiografia simples de abdome associada à história e exame físico compatíveis. Em oito pacientes, o diagnóstico pré-operatório foi confirmado por colonoscopia e em dois casos pelo toque retal.

Nos 23 pacientes com obstrução do cólon esquerdo, a idade mediana foi de 52 (39-84) anos, sendo $10(43,5 \%)$ do gênero masculino e $13(56,5 \%)$ do feminino. As operações realizadas nestes pacientes estão elencadas na Tabela 1. Em 14 (60,9\%) deles, o tratamento completo foi realizado em um só tempo, com ressecção e anastomose primária (grupo RAP), e em 9 (39,1\%), o tratamento foi programado com ressecção em estágios (grupo RE), realizando-se inicialmente colostomia, com ou sem ressecção da lesão obstrutiva.

\section{Operações realizadas}

As operações realizadas nos 14 pacientes tratados em um só tempo operatório (grupo RAP) foram as seguintes: a) ressecção segmentar e anastomose primária com PIOC $(n=10)$ e b) colectomia subtotal seguida de anastomose primária ileossigmóide ou ileorretal $(n=4)$. Todos os pacientes foram operados na posição de Lloyd-Davies sob anestesia geral. Basicamente, a técnica operatória utilizada foi a seguinte:
TABELA 1 - Tipo de operação realizada na obstrução do cólon esquerdo

\begin{tabular}{lcc}
\hline Tipo de operação & n & \% \\
\hline 1. Ressecção e anastomose & 14 & 60,9 \\
Ressecção segmentar + PIOC & 10 & 43,5 \\
Colectomia subtotal & 4 & 17,4 \\
2. Ressecção em estágios $\quad$ Hartmann (2 tempos) & 4 & 39,1 \\
Colostomia em alça (3 tempos) & 5 & 17,4 \\
Total & 23 & 100,0 \\
\hline
\end{tabular}

PIOC = Preparo intra-operatório do cólon

1. Ressecção segmentar com preparo intra-operatório do cólon - Nos 10 pacientes submetidos a este tipo de operação, o procedimento iniciou-se pela descompressão aspirativa do cólon com uma agulha associada a cateter plástico, tipo Jelco16 F. A seguir, mobilizou-se a flexura esplênica do cólon e removeuse o apêndice para introdução de um cateter Foley 20-24 F no ceco pelo coto apendicular. O cólon esquerdo foi seccionado com boa margem de segurança abaixo da lesão obstrutiva e, após as ligaduras do seu mesentério, foi mobilizado para fora da cavidade abdominal. Com cuidado e com auxílio de pinças de coproestase, realizou-se pequena colotomia de $2,5 \mathrm{~cm}$ logo acima da obstrução e, através dessa fenda, introduziu-se um tubo corrugado transparente de uso corriqueiro para gases anestésicos, que foi fixado com duas ou três ligaduras de linho 0 duplo. Pelo cateter Foley irrigou-se anterogradamente o cólon com 7 a $10 \mathrm{~L}$ de solução salina $0.9 \%$ aquecida a $38^{\circ} \mathrm{C}$, sendo o efluente captado por saco de lixo devidamente acoplado à porção terminal do tubo corrugado e colocado no nível inferior da mesa operatória. A irrigação só terminava quando o efluente estava claro e sem fezes. Nesse momento, irrigava-se pela última vez o cólon com solução fisiológica contendo $10 \%$ de polivinil pirrolidona iodo. A seguir, praticava-se a ressecção segmentar, sendo o coto retal irrigado também com a mesma solução através de anuscópio rígido ("rectal wash-out"). A anastomose foi realizada manualmente (plano único com pontos separados extramucosos com fios de poliglatina ou linho 3-0) em sete pacientes e com grampeador em três. O cateter Foley foi exteriorizado como cecostomia nos primeiros seis pacientes e foi retirado com fechamento em bolsa do coto apendicular nos últimos quatro. Nos pacientes que ficaram com a cecostomia no pós-operatório, esta era deixada aberta para descompressão, fechada quando o trânsito intestinal se normalizava e retirada após o $14^{\circ}$ dia pós-operatório. 
2. Colectomia subtotal - Nos quatro pacientes submetidos a esta operação, realizou-se, também de maneira inicial, descompressão do cólon com a utilização de agulha acoplada a cateter plástico tipo Jelco 16 e aspiração. A seguir, praticou-se a dissecção do cólon pelas goteiras parietocólicas, mobilização das flexuras hepática e esplênica e secção e ligadura dos vasos principais do mesocólon próximo à raiz. Realizou-se, então, a ressecção com margem de segurança abaixo da lesão obstrutiva e a aproximadamente 2-4 cm proximal à válvula ileocecal. A seguir, procedeu-se à irrigação do coto retal do modo anteriormente descrito e, finalmente, à anastomose manual primária término-terminal ileorretal ou ileossigmóide da forma já referida.

Dos nove pacientes restantes (grupo RE), quatro casos foram submetidos a ressecção segmentar e colostomia à Hartmann (ressecção em dois tempos) e cinco foram submetidos apenas a colostomia em alça no ângulo hepático (ressecção em três tempos).

Todos os pacientes receberam antibióticos por, no mínimo, 6 dias. O regime consistiu na associação de amicacina $500 \mathrm{mg}$ bid e metronidazol $500 \mathrm{mg}$ tds por via intravenosa. Na Tabela 2, encontramse as características clínicas e demográficas dos pacientes. Observouse distribuição bastante semelhante entre os dois grupos de tratamento, principalmente quanto ao estado nutricional avaliado pela albumina sérica e na avaliação da gravidade do caso clínico para a condução anestésica, determinada pelo grau ASA.

Para o seguimento dos pacientes no pós-operatório, foram registradas as seguintes variáveis: tempo total de internação, infecção de parede, deiscência da anastomose (fístula), complicações pulmonares e mortalidade. Nos pacientes do grupo RE, computaram-se os dois ou três atos operatórios que compunham o tratamento completo, sendo as complicações registradas no total por paciente por tratamento. Nesse grupo, registrou-se também o número de pacientes que não completou o tratamento.
Os dados foram apresentados como média \pm desvio padrão ou mediana (variação), conforme a distribuição dos dados ser homogênea ou não (teste de Levene).

\section{RESULTADOS}

Dois pacientes $(8,7 \%)$ faleceram no pós-operatório intrahospitalar. Ambos apresentaram complicações pulmonares, íleo prolongado e falência de múltiplos órgãos, pertenciam ao grupo RE e submeteram-se a operação de colostomia em alça. Não houve mortalidade entre pacientes operados no grupo RAP.

Quatro $(44,4 \%)$ dos pacientes no grupo RE não completaram o tratamento com o fechamento da colostomia. Dois, conforme descrito acima, faleceram no pós-operatório recente, e outros dois, um submetido a operação de Hartmann e outro a colostomia em alça, faleceram, respectivamente, 8 e 11 meses após a operação inicial, ainda com o estoma. No total, os nove pacientes deste grupo foram submetidos a 18 operações.

Treze $(56,5 \%)$ pacientes dentre os 23 iniciais evoluíram sem apresentar complicações. As complicações apresentadas pelos outros 10 pacientes $(43,5 \%)$ podem ser vistas, de acordo com o grupo, na Tabela 3. A incidência de complicações por paciente foi de $28,6 \%$ (4/ 14), no grupo ressecção e anastomose primária e de 66,7\% (6/9), no grupo submetido a ressecção em estágios.

Dentre os 14 casos operados com realização de anastomose primária, ocorreu um caso $(7,1 \%)$ de deiscência de anastomose seguida de fístula estercoral em paciente submetido a ressecção segmentar e PIOC. O paciente evoluiu com sepse pélvica importante, obrigando a realização de colostomia e drenagem do abscesso, tendo, então, evoluído favoravelmente. No grupo ressecção em estágios, ocorreram dois casos $(22,2 \%)$ de deiscência e fístula estercoral, ambos durante o fechamento de colostomias, sendo o primeiro em fechamento de colostomia

TABELA 2 - Variáveis clínicas e demográficas dos pacientes segundo a operação realizada

\begin{tabular}{|c|c|c|c|}
\hline \multirow[b]{2}{*}{ Variável } & & \multicolumn{2}{|c|}{ Tipo de operação } \\
\hline & & Ressecção e anastomose & Ressecção em estágios \\
\hline \multicolumn{4}{|l|}{ Gênero } \\
\hline & $\mathrm{F}$ & 9 & 4 \\
\hline & M & 5 & 5 \\
\hline Idade (anos)* & & $53(39-77)$ & $50(40-84)$ \\
\hline \multicolumn{4}{|l|}{ ASA } \\
\hline & II & 5 & 3 \\
\hline & III & 6 & 3 \\
\hline & IV & 3 & 3 \\
\hline Desnutrição** & & $5(35,7 \%)$ & $3(33,3 \%)$ \\
\hline
\end{tabular}

* = Mediana (variação)

** Albumina sérica $<3 \mathrm{mg} \%$ 
Hartmann e o segundo em colostomia em alça no terceiro tempo do tratamento em estágios. Os dois pacientes evoluíram bem com tratamento clínico e terapia nutricional enteral.

Sete $(30,4 \%)$ pacientes apresentaram supuração da parede abdominal, exigindo apenas curativos para o tratamento. Destes, três $(3 / 14 ; 21,4 \%)$ pertenciam ao grupo com ressecção e anastomose primária e quatro (4/9; $44,4 \%$ ) ao grupo com ressecção em estágios. O tempo de internação foi de 10 (6-45) dias, sendo de 15 (9-45) dias no grupo ressecção em estágios e de 8 (6-20) dias no grupo ressecção e anastomose.

Na Tabela 4 encontra-se o estádio dos casos pela classificação de Dukes. Nos dois que evoluíram para óbito, não foi possível o estádio pois os pacientes foram submetidos apenas a colostomia em alça e, assim, as peças não foram estudadas. Observou-se distribuição semelhante nos 21 casos possíveis de serem estudados em relação ao estádio mostrando, de maneira geral, tumores bastante avançados.

\section{DISCUSSÃO}

A presença de cólon distendido, edemaciado e repleto de fezes tem sido apontada como a principal causa de deiscência de anastomoses realizadas na vigência de obstrução do cólon esquerdo ${ }^{(1,11)}$. Porém, essa insegurança de realizar ressecção seguida de anastomose primária na presença de cólon mal preparado, vem sendo progressivamente menor entre os cirurgiões habituados às operações colorretais. Vários estudos clínicos comprovam que a ressecção segmentar, com auxílio do PIOC $^{(2,5,6,8,9,13,14,19,22)}$ ou a colectomia subtotal/total ${ }^{(4,10,12,23)}$, é segura e eficaz no tratamento da obstrução do cólon esquerdo. Experimentalmente, o uso do PIOC tem mostrado também ser vantajoso quanto à cicatrização ${ }^{(1,3)}$.

Nos 14 casos desta série, operados em um só tempo seja pelo uso de uma ou de outra técnica, não ocorreram óbitos e em apenas um caso houve deiscência da sutura anastomótica. De maneira geral, a morbidade observada foi menor quando a operação de ressecção e anastomose primária foi empregada. No entanto, este estudo é retrospectivo, com grupos heterogêneos para comparação e, por isso, os resultados devem ser vistos com cautela. Entretanto, o estádio do câncer, a classificação dos pacientes pelo grau ASA e a albumina sérica nos dois grupos foram muito semelhantes. Esses resultados confirmam as boas expectativas na experiência inicial do presente grupo com a técnica em um só tempo ${ }^{(2)}$.

TABELA 3 - Complicações pós-operatórias no tratamento da obstrução maligna do cólon esquerdo segundo o grupo

\begin{tabular}{lcc}
\hline & \multicolumn{2}{c}{ Tipo de operação } \\
\cline { 2 - 3 } Complicação & $\begin{array}{c}\text { Ressecção e anastomose } \\
(\mathrm{n}=14)^{*}\end{array}$ & $\begin{array}{c}\text { Ressecção em estágios } \\
(\mathrm{n}=9)^{* *}\end{array}$ \\
\hline Infecção de parede & $3(21,4 \%)$ & $4(44,4 \%)$ \\
Fístula & $1(7,1 \%)$ & $2(22,2 \%)$ \\
Trombose venosa & $1(7,1 \%)$ & $-(0,0 \%)$ \\
Pulmonar & $-(0,0 \%)$ & $3(33,3 \%)$ \\
Mortalidade & $-(0,0 \%)$ & $2(22,2 \%)$ \\
\hline
\end{tabular}

* = Quatro pacientes apresentaram cinco complicações

** $=$ Seis pacientes apresentaram 11 complicações

TABELA 4 - Estádio dos pacientes operados pela classificação de Dukes

\begin{tabular}{ccc}
\hline & \multicolumn{2}{c}{ Tipo de operação } \\
\cline { 2 - 3 } Estádio & $\begin{array}{c}\text { Ressecção e anastomose } \\
(\mathrm{n}=14)\end{array}$ & $\begin{array}{c}\text { Ressecção em estágios* } \\
(\mathrm{n}=9)\end{array}$ \\
\hline A & - & - \\
B & 5 & 3 \\
C & 5 & 3 \\
D & 4 & 1 \\
\hline
\end{tabular}

* Em dois casos não foi possível o estádio 
Nos nove pacientes operados pela técnica de ressecção em estágios, ocorreram dois óbitos e, em quase $45 \%$ dos casos, a colostomia não pôde ser fechada. Realmente, esta é uma das grandes críticas a esse tipo de operação, pois os casos de câncer com obstrução geralmente são avançados, o prognóstico é pior e paliativamente é melhor para o paciente que o tumor seja ressecado e que ele sobreviva sem estoma. Esses dados não causam surpresa, pois na literatura, de maneira geral, $16 \%$ a 54\% dos pacientes não completam o tratamento em estágios e acabam falecendo com a colostomia ${ }^{(8,16,18)}$. Além disso, há evidências que, a longo prazo, os resultados são mais favoráveis à ressecção e anastomose primária quando comparada à ressecção em estágios ${ }^{(7)}$.

Em muitos trabalhos comparativos entre o tratamento em um único tempo e em estágios, levou-se em conta, no segundo grupo, apenas a primeira operação ${ }^{(18)}$. Essa avaliação é distorcida, pois deveriam ser computados os dois ou três atos operatórios aos quais o paciente foi submetido para completar o tratamento. Quando isso é feito, os melhores resultados da operação em um só tempo ficam mais evidentes ${ }^{(7,12)}$. Nessa casuística, o tempo de internação total e a morbidade mostram que quando os dois ou três atos são vistos em conjunto, fica evidente que a resolução em um só tempo é melhor. No entanto, deve ficar claro que a manipulação de cólon obstruído é difícil e, assim, apenas cirurgiões treinados devem fazer essas operações. Caso o treinamento do cirurgião de plantão não seja adequado para tal, a colostomia pode trazer mais benefícios para o paciente.

Há, no momento, discussão na literatura a respeito da melhor técnica para o tratamento em um só tempo ${ }^{(21,24)}$. Ressecção segmentar e anastomose primária sem PIOC tem seus adeptos ${ }^{(17)}$, porém não tem sido a preferida pelos autores. Embora o preparo de cólon tenha sido questionado recentemente ${ }^{(20)}$, realizar anastomoses em cólon obstruído e sem preparo deve ser o temor da maioria dos cirurgiões. Dentro desse contexto, então, a preferência tem sido dada à ressecção segmentar auxiliada pelo PIOC e pela colectomia subtotal/total com anastomose ileorretal ou do sigmóide.
As principais vantagens do PIOC são a de oferecer cólon preparado para uma ressecção, permitir tratamento oncologicamente eficaz com ressecção colônica habitual e proporcionar melhor qualidade de vida para casos onde a ressecção foi paliativa ${ }^{(2,5,6,8,9,13,14,19,21,22)}$. Entretanto, o método envolve tempo operatório maior e há riscos de contaminação da cavidade abdominal com fezes durante a lavagem ${ }^{(23,24)}$. No entanto, essa situação não ocorreu em nenhum dos 10 casos operados desta série. Em outra experiência nacional com o PIOC ${ }^{(22)}$, isto também não ocorreu, havendo igualmente, relato de bons resultados.

Já a colectomia subtotal/total tem a vantagem de poder extirpar possíveis tumores sincrônicos e pólipos satélites do resto do cólon e permitir ressecção e anastomose primária com menor chance de contaminação da cavidade ${ }^{(4,10,12,23,24)}$. A principal desvantagem é a ressecção completa e desnecessária do cólon, podendo causar diarréia e incontinência para alguns pacientes ${ }^{(21,24)}$. A preferência do grupo tem sido para a ressecção associada ao PIOC, porém em alguns casos, optou-se pela colectomia subtotal, também com bons resultados.

Recentemente, o uso de próteses auto-expansivas tem sido relatado para o tratamento paliativo ou, ainda, como primeiro tempo no tratamento das obstruções do cólon esquerdo. Essa conduta tem ainda a vantagem de evitar operações desnecessárias em casos paliativos ${ }^{(15)}$. O papel dessas próteses precisa, ainda, ser melhor definido com novos estudos.

A análise global dos resultados mostrou mortalidade aceitável quando comparada com a literatura. Embora o presente trabalho seja retrospectivo, com casuística pequena, os achados mostraram morbidade alta e maior nos pacientes submetidos ao tratamento em estágios. Desta maneira, o conjunto dos resultados sugere que o tratamento em um só tempo na obstrução aguda do cólon esquerdo é seguro e pode ser indicado na maioria dos casos. Trabalhos prospectivos comparando a ressecção em tempo único e em estágios são, por conseguinte, recomendados.

Aguilar-Nascimento JE, Caporossi C, Nascimento M. Comparison between resection and primary anastomosis and staged resection in obstructing adenocarcinoma of the left colon. Arq Gastroenterol 2002;39(4):240-245.

ABSTRACT - Background - The surgical option to deal with obstruction of the left side of the large bowel is still controversial. Objective - To report the experience of our group in the treatment of malignant left-sided colonic obstruction focusing on the immediate results using either one-stage resection and primary anastomoses or staged resection. Patients/Methods - Twenty-three patients (median age $=52(39-84)$ years; 10 males and 13 females) with potentially resectable obstructed adenocarcinomas of the left colon entered the study. The patients were submitted to different surgical procedure: $14(60,9 \%)$ underwent one stage colonic resection (intra-operative lavage of colon $(\mathrm{n}=10)$ or subtotal colectomy $(\mathrm{n}=4)$; resection and primary anastomoses group) and 9 patients $(39,1 \%)$ underwent staged resection (Hartmann's operation $(n=4)$ or loop colostomy $(n=5)$; staged resection group). Results - Two patients $(8,7 \%)$ died. All were from the staged resection group. Four patients $(44,4 \%)$ of staged resection group did not complete the treatment with the closing of the colostomy. The incidence of complications was $28,6 \%$ in resection and primary anastomoses group (4/14) and 66,7\% in staged resection group (6/9). Hospital stay was 15 (9-45) in staged resection patients and 8 (6-20) in resection and primary anastomoses group. There was one case (7,1\%) of anastomotic dehiscence in resection and primary anastomoses group and two cases $(22,2 \%)$ in staged resection group. Conclusions - The treatment of obstruction of left colon in one stage is safe and may be indicated for the management of the majority of cases.

HEADINGS - Intestinal obstruction. Colorectal neoplasms. Colectomy. Colostomy. 


\section{REFERENCIAS BIBLIOGRÁFICAS}

1. Aguilar-Nascimento JE, Caporossi C, Figueiredo PC, Alves DC, Kobata CM, Chacon JC. The influence of peroperative irrigation of the obstructed left colon in the healing of primary anastomosis: an experimental study in rats. ABCD Arq Bras Cir Dig 1990;5:57-62.

2. Aguilar-Nascimento JE, Caporossi C, Marra JG, Freire EL. Ressecção e anastomose primária na obstrução neoplásica do cólon esquerdo com auxílio do preparo intestinal per-operatório. Rev Bras Coloproctol 1992;12:17-20.

3. Aguilar-Nascimento JE, Mathie RT, Man WK, Williamson RCN. Enhanced intraanastomotic healing by operative lavage with nutrient solutions in experimental left-sided colonic obstruction. Br J Surg 1995;82:461-4.

4. Arnaud J-P, Bergamaschi R. Emergency subtotal/total colectomy with anastomosis for acutely obstructed carcinoma of the left colon. Dis Colon Rectum 1994;37:685-8.

5. Chiappa A, Zbar A, Biella F, Staudacher C. One-stage resection and primary anastomosis following acute obstruction of the left colon for cancer. Am Surg 2000,66:619-22

6. Dudley HA, Radcliff AG, McGeehan D. Intraoperative irrigation of colon to permit primary anastomosis. Br J Surg 1980;67:80-1.

7. Fielding LP, Wells BW. Survival after primary and after staged resection for large bowel obstruction caused by cancer. Br J Surg 1974;61:16-8.

8. Forloni B, Reduzzi R, Paludetti A, Colpani L, Cavallari G, Frosali D Intraoperative colonic lavage in emergency surgical treatment of left-sided colonic obstruction. Dis Colon Rectum 1998;41:23-7.

9. Foster ME, Johnson CD, Billings PJ, Davies PW, Leaper DJ. Intraoperative antegrade lavage and anastomotic healing in acute colonic obstruction. Dis Colon Rectum 1986;29:255-9.

10. Hughes ES, Cuthbertson AM. Subtotal colectomy for obstructing carcinoma of the upper left colon. Dis Colon Rectum 1965;8:411-2.

11. Irvin TT, Goligher JC. Etiology of disruption of intestinal anastomosis. Br J Surg 1973;60:461-4.

12. Klatt GR, Martin WM, Gillespie JT. Subtotal colectomy with primary anastomosis without diversion in the treatment of obstructing carcinoma of the left colon. Am J Surg 1981;141:577-8.

13. Koruth NM, Krukowski ZH, Youngson GG, Hendry WS, Logie JR, Jones PF, Munro A. Intraoperative colonic irrigation in the management of left-sided large bowel emergencies. Br J Surg 1985;72:708-11.
14. Lee YM, Law Wl, Chu KW, Poon RT. Emergency surgery for obstructing colorectal cancers: a comparison between right-sided and left-sided lesions. J Am Coll Surg 2001;192:719-25.

15. Maritnez-Santos C, Lobato RF, Fradejas JM, Pinto I, Ortega-Deballon P, MorenoAzcoita M. Self-expandable stent before elective surgery vs. emergency surgery for the treatment of malignant colorectal obstructions: comparison of primary anatomosis and morbidity rates. Dis Colon Rectum 2002;45:401-6.

16. Monteiro MS, Barone B, Matos D. Resultados imediatos da operação em três tempos em doentes com neoplasia obstrutiva de cólon esquerdo. Rev Bras Coloproctol 1990;10:9-12

17. Naraynsingh V, Rampaul R, Maharaj D, Kuruvilla T, Ramcharan K, Pouchet B Prospective study of primary anastomosis without colonic lavage for patients with an obstructed left colon. Br J Surg 1999;86:1341-3.

18. Phillips RK, Hittinger R, Fry JS, Fielding LP. Malignant large bowel obstruction. Br J Surg 1985;72:296-302.

19. Pollock AV, Playforth MJ, Evans M. Peroperative lavage of the obstructed left colon to allow safe primary anastomosis. Dis Colon Rectum 1987;30:171-3.

20. Santos Jr JCM, Batista J, Sirimarco JT, Guimarães AS, Levy CE. Prospective randomised trial of mechanical bowel preparation in patients undergoing elective colorectal surgery. Br J Surg 1994,81:1673-6.

21. The Scotia Study Group. Subtotal Colectomy versus On-table Irrigation and Anastomosis. Single-stage treatment for malignant left-sided colonic obstruction: a prospective randomized clinical trial comparing subtota colectomy with segmental resection following intraoperative irrigation. $\mathrm{Br} \mathrm{J}$ Surg 1995;82:1622-7

22. Silva JH, Kerzner A, Formiga GLS, Matheus CO. Lavagem intestinal anterógrada transoperatória na obstrução neoplásica do cólon esquerdo. Rev Bras Coloproctol 1993; $13: 42-5$

23. Stephenson BM, Shandall AA, Farouk R, Griffith G. Malignant left-sided large bowel obstruction managed by subtotal/total colectomy. Br J Surg 1990;77:1098-102.

24. Torralba JA, Robles R, Parrilla P, Lujan JA, Liron R, Pinero A, Fernandez JA. Subtotal colectomy $v s$. intraoperative colonic irrigation in the management of obstructed left colon carcinoma. Dis Colon Rectum 1998;41:18-22.

Recebido em 4/3/2002 Aprovado em 22/10/2002. 